Изв. АН Эстонии. Физ. Матем., 1989, 38, № 3, 300-304

уДК 537.226 .4

Н. КРИСТОФЕЛЬ, А. ПИЩЕВ

\title{
О КОРРЕЛЯЦИОННЫХ СВОЙСТВАХ ЭЛЕКТРОННОЙ ПОДСИСТЕМЫ КРИСТАЛЛА СО СТРУКТУРНЫМ ФАЗОВЫМ ПЕРЕХОДОМ
}

\author{
(Представил В. Хиюняков)
}

Рассмотрены связанные состояния электрон-дырка, возникающие при вибронном структурном фазовом переходе типа смещения. Определены плотность конденсата таких пар и его суммарный импульс. Найдена корреляционная длина электронного параметра порядка.

1. Корреляционные свойства вибронного сегнетоэлектрика, связанные с фононным параметром порядка, в целях выяснения границ области подобия были исследованы в $\left.{ }^{1}\right]$. Ведущее структурный фазовый переход (ФП) межзонное электрон-фононное взаимодействие индуцирует также электронный параметр порядка - аномальные межзонные средние $\left[{ }^{2-5}\right]$, корреляционные свойства которого являются предмегом настоящего сообщения.

Гамильтониан рассматриваемой системы имеет вид $[2,3]$

$$
\begin{aligned}
H= & \sum_{\sigma, \mathbf{k}} \varepsilon_{\sigma}(\mathrm{k}) a_{\sigma \mathbf{k}}^{+} a_{\sigma \mathbf{k}}+N_{0}^{-1 / 2} \sum_{\sigma, \sigma^{\prime}}^{\prime \prime} \sum_{\mathbf{k}, \mathbf{q}} V_{\sigma \sigma^{\prime}}(\mathbf{q}) X \\
& X a_{\sigma \mathbf{k}}^{+} a_{\sigma^{\prime} \mathbf{k}-\mathbf{q}} y_{\mathbf{q}}+\frac{1}{2} \sum_{\mathbf{q}} M \hat{\omega}^{2} y_{\mathbf{q}} y_{-\mathbf{q}} .
\end{aligned}
$$

Здесь $\varepsilon_{\sigma}(\mathbf{k})$ - энергии электронных зон, $y_{\mathrm{q}}-$ нормальные координаты колебаний с массой $M$ и частотами $\hat{\omega}_{q}$, уже перенормированными фононными ангармонизмами $\hat{\omega}_{\mathbf{q}}=\omega_{\mathbf{q}}+\sum_{\mathbf{q}^{\prime}} A\left(\mathbf{q}, \mathbf{q}^{\prime}\right) \operatorname{coth} \hbar_{\omega_{\mathbf{q}^{\prime}}} / 2 k_{B} T$ $\left[{ }^{3}\right], V_{\sigma \sigma^{\prime}}(\mathbf{q})$ - константы электрон-фононного взаимодействия, $N_{0}-$ число элементарных ячеек в кристалле. При выполнении условия

$$
\sum_{\sigma, \sigma^{\prime}}^{\prime} \sum_{\mathbf{k}} \frac{\left|V_{\sigma \sigma^{\prime}}\left(\mathrm{q}_{0}\right)\right|^{2}}{N_{0} M \hat{\omega}_{\mathrm{q} 0}^{2}(T=0)}\left[\varepsilon_{\sigma^{\prime}}\left(\mathrm{k}+\mathrm{q}_{0}\right)-\varepsilon_{\sigma}(\mathrm{k})\right]^{-1}>1
$$

межзонное вибронное взаимодействие индуцирует структурный ФП типа смещения с образованием модулированной вектором $\mathbf{q}_{0}$ структуры (случай $\mathbf{q}_{0}=0$ соответствует сегнетоэлектрическому ФП) [ $\left.{ }^{3}\right]$.

2. Рассмотрим вопрос о возможности образования связанных состояний частиц при вибронном структурном фазовом переходе, и прежде всего - двухчастичных состояний. Для этого следует вычислить среднее число пар частиц $N_{2}$ в системе, описываемой гамнльтонианом (1), причем

$$
\begin{aligned}
N_{2}=\frac{1}{2}\left\langle\hat{N}^{2}-\hat{N}\right\rangle= & \frac{1}{2}\left\{\int\left\langle\psi^{+}\left(\mathbf{r}_{1}\right) \psi\left(\mathbf{r}_{1}\right) \psi^{+}\left(\mathbf{r}_{2}\right) \psi\left(\mathbf{r}_{2}\right)\right\rangle d \mathbf{r}_{1} d \mathbf{r}_{2}-\right. \\
& \left.-\int\left\langle\psi^{+}(\mathbf{r}) \psi(\mathbf{r})\right\rangle d \mathbf{r}\right\} .
\end{aligned}
$$


Здесь $\hat{N}$ - оператор числа частиц, $\psi(\mathbf{r})$ - электронные полёвыё операторы, выражающиеся с привлечением блоховских волновых функций $\varphi_{\sigma \mathbf{k}}(\mathbf{r})$ как

$$
\psi(\mathbf{r})=\sum_{\sigma, \mathbf{k}} \varphi_{\sigma \mathbf{k}}(\mathbf{r}) a_{\sigma \mathbf{k}}=\sum_{\sigma, \mathbf{k}} u_{\sigma \mathbf{k}}(\mathbf{r}) e^{i \mathbf{k r}} a_{\sigma \mathbf{k}},
$$

где операторы Ферми $a_{\sigma \mathrm{k}}$ относятся к зонным состояниям. В импульсном представлении с применением теоремы Вика из (3) и (4) получается

$$
N_{2}=\frac{1}{2} N^{2}-\frac{1}{2} \sum_{\sigma} \sum_{\mathbf{k}} f_{\sigma}^{2}(\mathrm{k})-\frac{1}{2} \sum_{\sigma, \sigma^{\prime}}^{\prime} \sum_{\mathbf{k}, \mathbf{q}}\left|\left\langle a_{\sigma \mathbf{k}}^{+} a_{\sigma^{\prime} \mathbf{k}-\mathbf{q}}\right\rangle\right|^{2},
$$

где $f_{\sigma}(\mathbf{k})$ - функция Ферми и средние типа $\left\langle a^{+} a^{+}\right\rangle$, $\langle a a\rangle$ положены равными нулю. Для аномальных межзонных средних $\left\langle a_{\sigma}^{+} a_{\sigma^{\prime}}\right\rangle \mid \sigma \neq \sigma^{\prime}$, согласно $\left[{ }^{3}\right]$, имеем

$$
\sqrt{N_{0}}\left\langle a_{\sigma \mathbf{k}}^{+} a_{\sigma^{\prime} \mathbf{k}-\mathbf{q}}\right\rangle=\frac{V_{\sigma \sigma^{\prime}}(\mathbf{q}) y_{\mathbf{q}}\left[f_{\sigma}(\mathbf{k})-f_{\sigma^{\prime}}(\mathbf{k}+\mathbf{q})\right]}{E_{\sigma}(\mathbf{k})-E_{\sigma^{\prime}}(\mathbf{k}+\mathbf{q})} \delta_{\mathbf{q}, \mathbf{q}_{0}},
$$

где $\mathbf{q}_{0}-$ волновой вектор - ведущих структурный ФП колебаний, $E_{\sigma}(\mathbf{k})$ - перенормированные вибронным взаимодействием энергии электронных зон. Последний член в (5) поделенный на объем кристалла $\vartheta$ описывает плотность числа связанных состояний вибронных электрон-дырочных пар $n_{0}$. Используя (6) для $n_{0}$ можно получить следующее выражение

$$
n_{0}=\sum_{\sigma, \sigma^{\prime}}^{\prime} \frac{\left|V_{\sigma \sigma^{\prime}}\left(\mathrm{q}_{0}\right) y_{\mathbf{q} 0}\right|^{2}}{2 N_{0} \vartheta} \sum_{\mathbf{k}} \frac{\left[f_{\sigma}(\mathbf{k})-f_{\sigma^{\prime}}\left(\mathbf{k}+\mathbf{q}_{0}\right)\right]^{2}}{\left[E_{\sigma}(\mathrm{k})-E_{\sigma^{\prime}}\left(\mathbf{k}+\mathrm{q}_{0}\right)\right]^{2}} .
$$

Для доказательства связанности двухчастичного состояния электрона и дырки ниже точки структурного ФП возьмем парциальную по отдельным зонам парную корреляционную функцию $G_{2}$, например,

$$
G_{2}^{(12)}\left(\mathbf{r}_{1}, \mathbf{r}_{2} ; \mathbf{r}_{1}^{\prime}, \mathbf{r}_{2}^{\prime}\right)=\left\langle\psi_{1}^{+}\left(\mathbf{r}_{1}\right) \psi_{2}\left(\mathbf{r}_{2}\right) \psi_{2}^{+}\left(\mathbf{r}_{2}^{\prime}\right) \psi_{1}\left(\mathbf{r}_{1}^{\prime}\right)\right\rangle
$$

Согласно принципу ослабления корреляций $\left[{ }^{6}\right]$

$$
G_{2}^{(12)}\left(\mathbf{r}_{1}, \mathbf{r}_{2} ; \mathbf{r}_{1}^{\prime}, \mathbf{r}_{2}^{\prime}\right) \rightarrow\left\langle\psi_{1}^{+}\left(\mathbf{r}_{1}\right) \psi_{2}\left(\mathbf{r}_{2}\right)\right\rangle\left\langle\psi_{2}^{+}\left(\mathbf{r}_{2}^{\prime}\right) \psi_{1}\left(\mathbf{r}_{1}^{\prime}\right)\right\rangle
$$

из (8) исключаются несвязанные состояния. После перехода к импульсному представлению и введения координат центра инерции $\mathbf{R}=\frac{1}{2}\left(\mathbf{r}_{1}+\mathbf{r}_{2}\right)$ и относительного движения $\mathbf{r}=\mathbf{r}_{2}-\mathbf{r}_{1}$ для (9) получается

$$
\begin{aligned}
G_{2}^{(12)} \rightarrow & \sum_{\mathbf{k}, \mathbf{k}^{\prime}} \sum_{\mathbf{q}, \mathbf{q}^{\prime}}\left\langle a_{1 \mathrm{k}+\mathbf{q} / 2}^{+} a_{2 \mathrm{k}-\mathrm{q} / 2}\right\rangle\left\langle a_{1 \mathbf{k}^{\prime}+\mathbf{q}^{\prime} / 2}^{+} a_{2 \mathbf{k}^{\prime}-\mathbf{q}^{\prime} / 2}\right\rangle^{*} X \\
& \quad \times \chi_{12 \mathrm{k}, \mathbf{q}}^{*}(\mathbf{r}, \mathbf{R}) \chi_{12 \mathbf{k}^{\prime}, \mathbf{q}^{\prime}}\left(\mathbf{r}^{\prime}, \mathbf{R}^{\prime}\right) e^{-i \mathbf{q} \mathbf{R}} e^{i \mathbf{q}^{\prime} \mathbf{R}^{\prime}},
\end{aligned}
$$

где

$$
\chi_{12 \mathrm{k}, \mathbf{q}}^{*}(\mathbf{r}, \mathbf{R})=u_{1 \mathrm{k}+\mathrm{q} / 2}^{*}(\mathrm{R}-\mathrm{r} / 2) u_{2 \mathrm{k}-\mathbf{q} / 2}(\mathbf{R}+\mathbf{r} / 2) e^{i \mathrm{kr}}
$$

и выражение для $\chi_{12} \mathbf{k}^{\prime} \mathbf{q}^{\prime}\left(\mathbf{r}^{\prime}, \mathbf{R}^{\prime}\right)$ комплексно сопряжено (11) с заменой нештрихованных аргументов штрихованными. Учтем теперь, что аномальные межзонные средние, фигурирующие в (10) отличны от нуля только для активного в структурном ФП волнового вектора q т. е. $\mathbf{q}, \mathbf{q}^{\prime}=\mathbf{q}_{0}$. Окончательно имеем 


$$
\begin{aligned}
G_{2}^{(12)} & \rightarrow \sum_{\mathbf{k}, \mathbf{k}^{\prime}}\left\langle a_{1 \mathbf{k}+\mathbf{q}_{0} / 2}^{+} a_{2 \mathbf{k}-\mathbf{q}_{0} / 2}\right\rangle\left\langle a_{1 \mathbf{k}^{\prime}+\mathbf{q}_{0} / 2}^{+} a_{2 \mathbf{k}^{\prime}-\mathbf{q}_{0} / 2}\right\rangle^{*} X \\
& \times \chi_{12 \mathbf{k}, \mathbf{q}_{0}}^{*}(\mathbf{r}, \mathbf{R}) \chi_{12 \mathbf{k}^{\prime}, \mathbf{q}_{0}}\left(\mathbf{r}^{\prime}, \mathbf{R}^{\prime}\right) \mathrm{e}^{-i \mathbf{q}_{0}\left(\mathbf{R}-\mathbf{R}^{\prime}\right)} .
\end{aligned}
$$

На основании (12), учитывая (6) и согласно $\left[{ }^{7}\right]$, можно считать, что в температурной области, где аномальные средние отличны от нуля, (ниже $T_{C}$ ) имеется конденсат из связанных вибронным взаимодействием пар электрон-дырка с суммарным импульсом $\hbar q_{0}$, равным импульсу активного в структурном ФП поперечного оптического колебания. При этом

$$
\Phi_{12}(\mathbf{r}, \mathbf{R})=\left\langle\psi_{1}^{+}\left(\mathbf{r}_{1}\right) \psi_{2}\left(\mathbf{r}_{2}\right)\right\rangle=\sum_{\mathbf{k}} \chi_{12 \mathrm{k}, \mathbf{q}_{0}}^{*}(\mathbf{r}, \mathbf{R}) e^{-i \mathbf{q}_{0} \mathbf{R}}\left\langle a_{1 \mathbf{k}+\mathbf{q}_{0} / 2}^{+} a_{2 \mathbf{k}-\mathbf{q}_{0} / 2}\right\rangle
$$

есть волновая функция связанной пары электрон-дырка.

Для сегнетоэлектрика $\left(\mathbf{q}_{0}=0\right)$ при нулевой температуре

$$
\Phi_{12}(\mathbf{r}, \mathbf{R}) \simeq \frac{V_{12}(0) y_{00}}{2 \pi^{2} \sqrt{N_{0}}} \frac{\chi_{120,0}^{*}(\mathbf{r}, \mathbf{R}) \lambda(r)}{r}
$$

где

$$
\lambda(r)=\int_{0}^{h_{0}} \frac{k \sin k r}{E(k)+\Delta} d k \text {. }
$$

Здесь мы перешли от суммирования по $k$ к интегрированию, причем провели усреднение по углам. Для упрощения вибронно перенормированные энергии зон заменены своими затравочными значениями $\varepsilon_{1}(\mathbf{k})$ и $\varepsilon_{2}(\mathbf{k})$, полагаемые изотропными и параболическими с эффективными массами $m_{1}$ и $m_{2}, E(k)=\frac{\hbar^{2} k^{2}}{\mu}, \mu^{-1}=\frac{1}{2}\left(m_{1}^{-1}+m_{2}^{-1}\right), k_{0}=\left(\frac{6 \pi^{2} N_{0}}{\mho}\right)^{1 / 3}$ и $\Delta$ - диэлектрическая щель. Интеграл (15) преобразуется к виду

$$
\lambda(r)=\frac{\mu}{\hbar^{2}}\left\{S i\left(k_{0} r\right)-\frac{\pi}{2}\left(1-e^{-r / \xi^{\prime}}\right)+\Delta \int_{k_{0}}^{\infty} \frac{\sin k r d k}{k[E(k)+\Delta]}\right\},
$$

где интегральный синус $S i(t)=\int_{0}^{t} \frac{\sin z}{z} d z$. Величина $\xi^{\prime}=\hbar / \sqrt{\mu \Delta}$ имеет смысл эффективного расстояния между электроном и дыркой в $r$-пространстве. В самом деле, электрон и дырка, разделены в Г-точке энергетической щелью $\Delta$, которой в свою очередь соответствует эффективный волновой вектор носителя $k^{\prime}=\sqrt{\mu \Delta} / \hbar$. Обратная величина $k^{\prime}$ и определяет $\xi^{\prime}$.

Первые два слагаемых в (16) меняются на длине порядка $\xi^{\prime}$. Считая $k_{0}>k^{\prime}$, при $r>\xi^{\prime} \quad S i\left(k_{0} \xi^{\prime}\right) \rightarrow \frac{\pi}{2}$ и функция $S i\left(k_{0} r\right)-$ $-\frac{\pi}{2}+\frac{\pi}{2} \exp \left(-r / \xi^{\prime}\right)$ убывает экспоненциально. С использованием известных асимптотических разложений интегрального снлуса [ $\left.{ }^{8}\right]$

$$
\int_{k_{0}}^{\infty} \frac{\sin k r d k}{k[E(k)+\Delta]} \simeq \frac{1}{k_{0}^{3} r}\left\{\cos k_{0} r+\frac{3 \sin k_{0} r}{k_{0} r}\right\}
$$

так что для $\lambda(r)$ при $r>\xi^{\prime}$ имеем

$$
\lambda(r) \simeq \frac{\mu}{\hbar^{2}}\left[\frac{\pi}{2} e^{-r / \xi^{\prime}}+\frac{1}{k_{0}^{3} r}\left\{\cos k_{0} r+\frac{3 \sin k_{0} r}{k_{0} r}\right\}\right] .
$$


Следовательно, осцилляционная зависимость в пределах элементарной ячейки и затухание на расстояниях порядка нескольких элементарных ячеек произведения блоховских амплитуд $\chi_{120,0}^{*}(\mathbf{r}, \mathbf{R})$ модулируются экспоненциально - убывающей и осцилляционно-затухающей на длинах $r>\xi^{\prime}$ функцней $\lambda(r)$, что в результате и определяет поведение волновой функции связанной электрон-дырочной пары $\Phi_{12}(\mathbf{r}, \mathbf{R})$. Это подтверждает интерпретацию длины $\xi^{\prime}$ как меры протяженности связанной вибронным взаимодействием электрон-дырочной пары. Для сегнетоэлектрика введением средней щели $\bar{\Delta}$ при нулевой температуре из (7) получаем

$$
n_{0} \simeq n\left[\frac{V_{12}(0) y_{00}}{\bar{\Delta} \sqrt{N_{0}}}\right]^{2}
$$

где $n=N / \vartheta$ - концентрация электронов в кристалле. Оценки для широкощельных сегнетоэлектриков типа $\mathrm{BaTiO}_{3}$ дают $n_{0} / n \sim 10^{-2}$, а для сегнетоэлектриков-полупроводников $\mathrm{A}^{\mathrm{IV}} \mathrm{B}^{\mathrm{VI}} n_{0} / n \sim 10^{-3}-10^{-4}$.

С приближением к точке ФП второго рода $y_{00} \rightarrow 0$ и $n_{0}$ исчезают. При ФП первого рода $n_{0}$ аналогично $y_{00}$ скачком обращается в ноль. Отсюда следует принципиальная возможность объяснения микроскопической природы электронной скрытой теплоты перехода. Так, например, при сегнетоэлектрическом ФП первого рода из сегнетофазы в парафазу для диссоциации оставшихся связанными в окрестности точки перехода электрон-дырочных пар требуется затратить дополнительную энергию, равную суммарной энергии связи $\sim N V_{12}^{2}(0) y_{00}^{2} / N_{0} \bar{\Delta}$ непродиссоциировавших к началу ФП связанных электрон-дырочных пар. Для обратного перехода ситуация меняется в противоположную сторону - образование макроскопического числа связанных электрон-дырочных пар энергетически выгодно, так что избыточная энергия выделяется кристаллом.

3. При наличии флуктуаций $y_{0} q / \sqrt{N_{0}}$ относительно низкосимметричного искажения решетки $y_{00} / \sqrt{N_{0}}$ аномальные межзонные средние приобретают зависимость от q (в коэрдинатном представлении от $\mathbf{R})$. Соответствующая корреляционная фуккция $\Gamma_{\mathbf{q}}$ в импульсном представлении пропорциональна $\sum_{\sigma, \sigma^{\prime}}^{\prime} \sum_{\mathbf{k}}\left\langle a_{\sigma \mathrm{k}}^{+} a_{\sigma^{\prime} \mathbf{k - q}}\right\rangle$. Выясним соотношение электронной и фононной коррелящионных длин. Удобно применить методический прием, рассмотренный в [ $\left.{ }^{9}\right]$, определив корреляционную длину положением ведущей сингулярности соответствующей корреляционной функции. В нашем случае для высогосимметричной фазы

$$
\xi_{e}^{2}=-\left.\frac{d \Gamma_{\mathrm{q}} / d \mathrm{q}^{2}}{\Gamma_{\mathrm{q}}}\right|_{\mathrm{q} \rightarrow 0}
$$

С учетом уравнения самосогласования для $y_{0 q}$ из (19)

$$
\hat{\omega \omega}_{\mathrm{q}}^{2} y_{0 \mathbf{q}}=-N_{0}^{-1 / 2} \sum_{\sigma, \sigma^{\prime}}^{\prime} V_{\sigma \sigma^{\prime}}(\mathbf{q}) \sum_{\mathbf{k}}\left\langle a_{\sigma \mathbf{k}}^{+} a_{\sigma^{\prime} \mathbf{k}-\mathbf{q}}\right\rangle
$$

пользуясь зависимсгтью $V_{12}^{2}(q)=V_{12}^{2}(0)\left(1-d^{2} q^{2}\right)$, где $d-$ постоянная решетки, и обозначая $\Delta_{1}(\mathrm{k}, \mathrm{q})=\varepsilon_{2}(\mathrm{k}+\mathrm{q})-\varepsilon_{1}(\mathrm{k}), \quad \Delta_{2}(\mathrm{k}, \mathrm{q})=$ $=\varepsilon_{2}(\mathbf{k})-\varepsilon_{1}(\mathrm{k}+\mathrm{q})$, находим 


$$
\begin{gathered}
\xi_{e}^{2}=\xi_{p h}^{2}+\frac{1}{2} d^{2}- \\
-\left[\sum_{k} \frac{2}{\Delta_{1}(\mathrm{k})}\right]^{-1}\left\{\frac{\partial}{\partial \mathrm{q}^{2}} \sum_{\mathrm{k}}\left[\Delta_{1}^{-1}(\mathrm{k}, \mathrm{q})+\Delta_{2}^{-1}(\mathrm{k}, \mathrm{q})\right]\right\}_{\mathrm{q} \rightarrow 0},
\end{gathered}
$$

где $\xi_{p h}-$ изученная в $\left[{ }^{1}\right]$ длина когерентности фононного параметра порядка в парафазе. Последний член в (21) можно аппроксимировать как $\hbar^{2} / \mu \bar{\Delta}$. Определение (19) можно распространить и на область сегнетофазы. Тогда под $\xi_{p h}$ следует понимать длину когерентности фононного параметра порядка в сегнетофазе, а последние два слагаемых в (21) следует разделить на два. Следовательно, масштабы изменения электронного и фононного параметров порядка практически совпадают. Пользуясь знанием $\xi_{p h}(T=0)$, из [ $\left.{ }^{1}\right]$ получаем, что электрондырочные пары разнесены в координатном пространстве на расстояния $\sim 20 \AA$, т. е. на расстояния около нескольких постоянных решетки.

\section{Л ИТ Е Р А Т Р А}

1. Кристофель Н.. Пищев А. // Изв. АН ЭССР. Физ. Матем., 1986, 35, № 2, $157-162$.

2. Kristoffel, N. N., Konsin, P. J. // Ferroelectrics, 1978, 21, № 1-4, 477-479.

3. Konsin, P. // Phys. stat. sol. (b), 1978, 86, № 1, 57-66.

4. Гирибере Я.Г., Тамарченко В. Н. // Физ. твердого тела, 1976, 18, № 3, 10661072.

5. Гиршберг Я. Г., Тамарченко В. Н. // Физ. твердого тела, 1976, 18, № 11, 33403347.

6. Боголюбов H. Н., Боголюбов Н. Н. (мл.) Введение в квантовую статистическую механику. М., Наука, 1984.

7. Свидзинский A. B. Пространственно-неоднородные задачи теории сверхпроводимости. М., Наука, 1982.

8. Справочник по специальным функциям (Ред. М. Абрамович, И. Стиган). М., Наука, 1979.

9. Вильсон К., Когут Дж. Ренормализационная группа и в-разложение. М., Мир, 1975 .

Институт физики

Академии наук Эстонской ССР

Поступила в редакцию 20/VI 1988

\section{N. KRISTOFFEL, A. PISTSEV}

\section{STRUKTUURSE FAASISIIRDEGA KRISTALLI ELEKTRONALASUSTEEMI KORRELATSIOONIOMADUSTEST}

On käsitletud elektroni ja augu seotud seisundeid, mis tekivad nihke tüüpi vibroonse struktuurse faasisiirde korral, ning määratud selliste paaride kondensaadi tihedus ja summaarne impulss. On leitud elektroonse korrastusparameetri korrelatsioonipikkus.

\section{N. KRISTOFFEL and A. PISHCHEV}

\section{ON THE CORRELATION PROPERTIES OF AN ELECTRONIC SUBSYSTEM OF A CRYSTAL WITH THE DISPLACIVE STRUCTURAL PHASE TRANSITION}

Coupled electron-hole states arising in the vibronic displacive structural phase transition are considered. The density of the condensate of such pairs and its net momentum are determined. The correlation length of the electronic order parameter is found. 